\title{
CISTOSTOMIA COM SONDA DE FOLEY PARA DESVIO TEMPORÁRIO DO FLUXO URINÁRIO EM FELINO ACOMETIDO POR RUPTURA URETRAL IATROGÊEICA
}

\author{
Juliana de Abreu Pereira ${ }^{1}$ \\ Acácia Romão Pereira das Neves ${ }^{2}$ \\ Patricia de Oliveira ${ }^{3}$ \\ Ana Carolina de Oliveira Almeida ${ }^{3}$ \\ Chaiene Souza Soares ${ }^{3}$ \\ Katherinne Barth Wanis Figueiredo ${ }^{4}$
}

\begin{abstract}
RESUMO
A ruptura uretral iatrogênica geralmente é uma intercorrência secundária à passagem forçada de sondas rígidas durante a tentativa de desobstrução uretral; procedimento este realizado como forma de eliminação imediata da urina em casos de retenção aguda. Pacientes obstruídos podem manifestar sinais de injúria renal aguda, sendo necessário o pronto restabelecimento do fluxo urinário. O presente estudo tem como objetivo demonstrar o uso de uma técnica de cistostomia temporária em felino com ruptura de uretra, feito a partir da inserção de uma sonda tipo Foley na região ventral da bexiga e fixação desta à parede abdominal para que a urina pudesse ser conduzida ao meio externo.
\end{abstract}

Palavras-chave: uroabdome, iatrogenia, gato.

\section{CYSTOSTOMY WITH FOLEY TUBE FOR TEMPORARY DEVIATION OF URINARY FLOW IN FELINE AFFECTED BY IATROGENIC URETHRAL RUPTURE}

\begin{abstract}
The iatrogenic urethral rupture is usually an intercurrence secondary to the forced passage of rigid tubes during the urethral clearance attempt; procedure that is performed as a means of immediate elimination of urine in cases of acute retention. Obstructed patients may show signs of acute kidney injury, requiring prompt restoration of urinary flow. The present study aims to demonstrate the use of a temporary cystostomy technique in feline with rupture of the urethra, made from the insertion of a Foley type probe in the ventral region of the bladder and fixation of it to the abdominal wall so that the urine could be conducted to the external environment.
\end{abstract}

Key words: uroabdomen, iatrogeny, cat.

\footnotetext{
${ }^{1}$ Docente do curso de Medicina Veterinária da Universidade Sociedade Educacional de Santa Catarina (UNISOCIESC), campus Joinville. juliana.abreu@unisociesc.com.br.

2 Médica Veterinária; pós-graduada em Patologia Clínica Animal pelo Instituto Qualittas de Pós Graduação (RJ)

${ }^{3}$ Médica Veterinária autônoma, com Residência em Medicina Veterinária, área de concentração Clínica Médica de Animais de Companhia, UFRRJ, Seropédica, RJ.

${ }^{4}$ Discente do curso de Medicina Veterinária da Universidade Sociedade Educacional de Santa Catarina (UNISOCIESC), campus Joinville.
}

Pereira JA, Neves ARP, Oliveira P, Almeida ACO, Soares CS, Figueiredo KBW. Cistostomia com sonda de Foley para desvio temporário do fluxo urinário em felino acometido por ruptura uretral iatrogênica. Vet. e Zootec. 2020.; 2: 001-007. 


\title{
CISTOSTOMÍA CON TUBO DE FOLEY PARA LA DESVIACIÓN TEMPORAL DEL FLUJO URINARIO EN FELINO AFECTADO POR RUPTURA URETRAL IATROGÉNICA
}

\begin{abstract}
RESUMEN
La ruptura uretral iatrogénica suele ser una intercurrencia secundaria al paso forzado de tubos rígidos durante el intento de aclaramiento uretral; procedimiento que se realizó como un medio de eliminación inmediata de orina en casos de retención aguda. Los pacientes obstruidos pueden mostrar signos de lesión renal aguda, lo que requiere una pronta restauración del flujo urinario. El presente estudio tiene como objetivo demostrar el uso de una técnica de cistostomía temporal en felinos con ruptura de la uretra, realizada mediante la inserción de una sonda tipo Foley en la región ventral de la vejiga y su fijación a la pared abdominal para que la orina pueda ser conducida al ambiente externo.
\end{abstract}

Palabras clave: uroabdomen, iatrogenia, gato.

\section{INTRODUÇÃO}

A síndrome denominada Doença do Trato Urinário Inferior do Felino (DTUIF), caracterizada por combinação das enfermidades cistite e uretrite, frequentemente culmina na ocorrência de obstrução uretral. A anatomia peculiar da porção peniana da uretra de felinos machos, com uma leve curvatura, favorece a ocorrência de obstruções, seja por urólitos ou por tampões mucosos oriundos de processos inflamatórios (1-4).

Mudanças na composição de rações foram fundamentais para diminuir acentuadamente a formação de urólitos, apesar de não diminuir a incidência da doença, que é caracterizada por ser crônica e apresentar recidivas em algumas situações, principalmente de estresse $(2,5)$. Atualmente, nota-se que a manutenção de um pH urinário ácido é mais importante do que propriamente dito o controle da ingestão de minerais em quantidades reduzidas para que haja uma formação menor de cálculos. A maioria dos casos de DTUIF ocorre em machos, entre $1 \mathrm{e}$ 4 anos, castrados, embora fêmeas também possam ser afetadas.

Felinos obstruídos frequentemente apresentam como sinais disúria, associada ou não à hematúria, estrangúria, vômitos em decorrência de uremia, e cistite secundária à retenção urinária. A terapêutica implica na desobstrução uretral do paciente, bem como terapia de suporte, através de fluidoterapia, antibioticoterapia, além do uso de anti-inflamatórios, ainda que o protocolo possa não beneficiar alguns pacientes (6). O procedimento de desobstrução deve ser realizado delicadamente, e sem pressa, a fim de evitar a ocorrência de ruptura iatrogênica da uretra $(1,7)$.

O tratamento é variado, pois depende do grau e do tempo de duração da obstrução. Mesmo que alguns procedimentos clínicos - desobstruções uretrais - auxiliem na eliminação imediata da urina, aproximadamente metade dos casos apresenta recidivas nos seis meses seguintes. Caso não seja possível realizar a desobstrução uretral, deve-se recorrer à uretrostomia (8) pois, caso a obstrução uretral não seja resolvida, o animal pode vir a óbito em 72 horas.

Pereira JA, Neves ARP, Oliveira P, Almeida ACO, Soares CS, Figueiredo KBW. Cistostomia com sonda de Foley para desvio temporário do fluxo urinário em felino acometido por ruptura uretral iatrogênica. Vet. e Zootec. 2020.; 2: 001-007. 
O presente relato tem por objetivo descrever uma técnica de desvio urinário temporário, em felino com ruptura de uretra, que possibilitou a melhoria das condições clínicas do paciente antes da realização de uma uretrostomia definitiva.

\section{RELATO DE CASO}

Um felino, Pelo Curto Brasileiro, macho, de aproximadamente 1 ano e 4 meses de idade foi atendido em Clínica Veterinária da região metropolitana do Rio de Janeiro, transferido de outra clínica, com quadro de obstrução uretral. Segundo o proprietário, o animal estava em tratamento para DTUIF por dois meses; porém, nos últimos dias, apresentava-se apático, inapetente e com perda de peso. Durante a anamnese, foi observado grau de desidratação estimado em $8 \%$, mucosas hipocoradas e baixo escore corporal. $\mathrm{O}$ animal apresentava o terço distal da uretra peniana dilacerado, porém já em processo de cicatrização, além de demonstrar sensibilidade aumentada à palpação e abdome abaloado. Procedeu-se fluidoterapia, administração de ceftriaxona $1 \mathrm{~g}$ (30mg/Kg, por via intravenosa - IV - a cada 12 horas - BID), meloxicam (Maxican ${ }^{\circledR}$ 0,2\%; na dose de $0,1 \mathrm{mg} / \mathrm{Kg}$, IV, a cada 24 horas - SID) e a desobstrução uretral através da sonda flexível tipo Tom Cat ${ }^{\circledR}$, porém, sem sucesso. No mesmo dia de entrada do paciente, o mesmo foi submetido à cistocentese para esvaziamento do conteúdo vesical, procedimento este que foi repetido no dia seguinte à entrada.

Diante do quadro debilitante do animal e do indício de ruptura iatrogênica da uretra e risco iminente de ruptura vesical com extravasamento de urina para a cavidade abdominal, optou-se pela intervenção cirúrgica emergencial para desvio do fluxo urinário, na tentativa de dar tempo hábil para o reestabelecimento das condições clínicas adequadas para a realização da uretrostomia.

O animal foi tranquilizado, por via intravenosa, com associação de maleato de acepromazina $(0,05 \mathrm{mg} / \mathrm{Kg})$, cloridrato de midazolam $(0,2 \mathrm{mg} / \mathrm{Kg})$ e cloridrato de cetamina (2 $\mathrm{mg} / \mathrm{Kg}$ ). Em seguida, foi submetido à tricotomia ampla da região ventral do abdome da cicatriz umbilical até a pelve. Foi realizada a indução anestésica por via intravenosa com propofol $(6 \mathrm{mg} / \mathrm{Kg})$, o que possibilitou a intubação do paciente; e manutenção com isoflurano e oxigênio $100 \%$ em sistema semi-aberto. Por todo período trans-cirúrgico, o animal recebeu fluidoterapia com cloreto de sódio $0,9 \%(50 \mathrm{~mL} / \mathrm{Kg} / \mathrm{h})$ por via intravenosa.

Procedeu-se uma celiotomia mediana medindo aproximadamente 1,5 a 2 centímetros da margem púbica cranial, a fim de se investigar toda a cavidade abdominal. Ao acessar a cavidade, foi encontrada grande quantidade de urina livre; e a vesícula urinária encontrava-se íntegra e retraída, com evidências de ruptura parcial da uretra próximo ao colo vesical. Foi realizada a drenagem do líquido livre, lavagem da cavidade utilizando solução físiológica 0,9\% morna; e após incisão na bexiga na sua face ventral, houve inserção de sonda tipo Foley siliconizada Rusch Amber ${ }^{\circledR}$, de duas vias, tamanho 18. Após posicionamento da sonda dentro da vesícula urinária, o cuff foi inflado e a sonda foi fixada na parede abdominal com fio absorvível de média duração Poliglactina 910 (Vicryl ${ }^{\circledR}$ ) de espessura 3-0 não cortante, utilizando-se sutura tipo descontínua simples. Com a fixação adequada da sonda, foi realizada a lavagem de toda cavidade com solução de cloreto de sódio $0,9 \%$ aquecida, com posterior sutura da parede abdominal e fixação da sonda para saída da urina para o exterior do corpo. A sutura externa empregada foi do tipo dedo chinês, sendo feita com fio de Nylon (Ethicon ${ }^{\circledR}$ ) de espessura 3-0. Imagens do trans-cirúrgico estão demonstradas na Figura 1. 


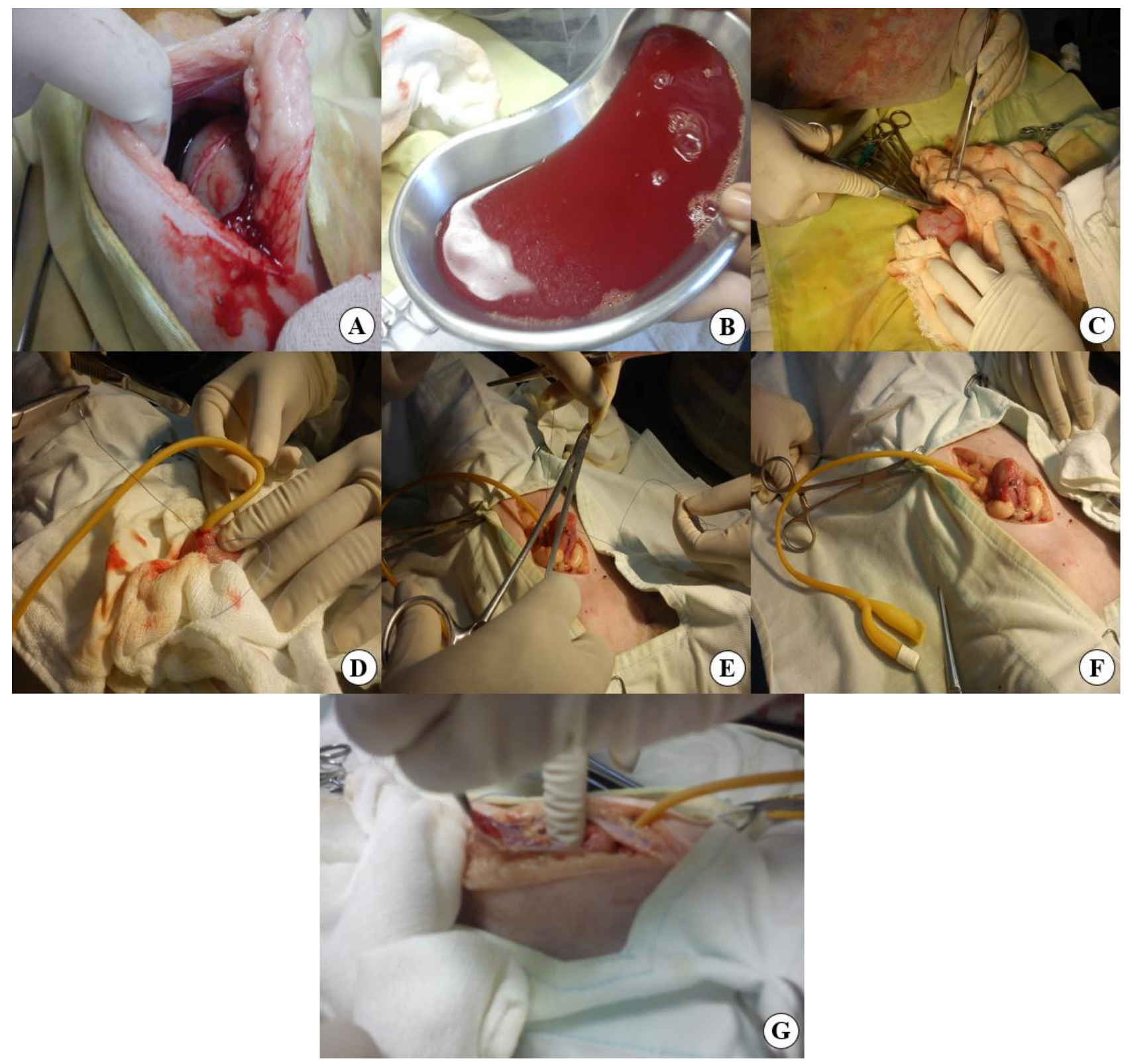

Legenda: A - celiotomia mediana para exploração da cavidade abdominal; B - líquido livre obtido do abdome, com aspecto avermelhado; C - realização da cistostomia; D - implantação da sonda Foley na vesícula urinária; E

- fechamento da incisão da cistostomia; F - avaliação do escoamento de urina através da sonda Foley; G fechamento da cavidade abdominal com fixação da sonda na parede abdominal

Figura 1. Imagens do trans-cirúrgico de cistostomia do paciente citado

O animal permaneceu com bandagem abdominal com atadura durante todo período pósoperatório, sendo realizados curativos diários com solução fisiológica e pomada de colagenase e cloranfenicol (Kollagenase ${ }^{\circledR}$ pomada); sendo mantida a fluidoterapia intravenosa, antibioticoterapia anterior com associação de enrofloxacino 2,5\% (5 mg/kg, por via subcutânea - SC, SID); cloridrato de tramadol $(1 \mathrm{mg} / \mathrm{kg}$, por via intramuscular - IM, a cada 8 horas - TID) e cloridrato de petidina. No dia seguinte ao procedimento, o animal 
voltou a aceitar alimentação forçada na seringa com dieta hiperproteica (Hill's A/D $\left.{ }^{\circledR}\right)$; passando, logo em seguida, a ingerir água e ração seca de forma espontânea. $\mathrm{O}$ fluxo urinário manteve-se constante e com escoamento por gravidade. Passados oito dias do procedimento de desvio urinário, o animal foi submetido à uretrostomia para desvio permanente do fluxo urinário; recebendo alta quatro dias depois. Esta técnica consistiu na realização de sutura do ponto de ruptura da vesícula urinária com o cólon, utilizando sutura tipo descontínua simples com fio absorvível de média duração Poliglactina 910 (Vicryl ${ }^{\circledR}$ ) de espessura 3-0 não cortante. Quinze dias após a uretrostomia definitiva, o paciente mostrou-se clinicamente sadio; com eliminação da urina através do trato digestório como esperado; e sem complicações pós-operatórias.

\section{DISCUSSÃO}

A DTUIF é uma enfermidade de grande importância na medicina felina, principalmente quando observada a prevalência desta doença em felinos machos (6) e submetidos à alimentação seca industrializada (9).

Como descrito para o felino deste quadro clínico, o insucesso nos procedimentos terapêuticos não-cirúrgicos decorreu da prévia ocorrência de ruptura de um segmento uretral por tentativa anterior de desobstrução de maneira inadequada. A incapacidade de eliminação de urina por parte do paciente, bem como a ruptura da uretra em porção média da mesma, culminou na instalação de quadro de uroabdome, o qual conduziria o paciente ao óbito em aproximadamente 72 horas, em decorrência de uremia severa. O emprego da cistocentese como manejo terapêutico para redução do volume vesical não foi suficientemente capaz de manter o paciente com níveis de compostos nitrogenados toleráveis, o que pode ser observado pela piora clínica do felino, mesmo após realização do procedimento por duas vezes.

A laparotomia exploratória realizada foi de extrema importância para determinação final dos fatores predisponentes do uroabdome, o que reitera que este procedimento é uma importante ferramenta diagnóstica para casos onde exista suspeita de ruptura uretral, sem evidências nos exames de imagem. Além disso, a abertura da cavidade abdominal possibilitou a realização de uma cistostomia temporária que foi determinante na recuperação do animal, e que possibilitou uma melhora clínica adequada antes do ato cirúrgico posterior e definitivo. $\mathrm{O}$ retorno à alimentação e ingestão hídrica por parte do animal no dia seguinte à colocação da sonda confirmam a recuperação clínica do mesmo no pós-operatório mediato.

A cistostomia empregada resultou em êxito apenas por não haver ruptura da vesícula urinária, cuja ocorrência impossibilitaria a utilização da técnica, implicando em realização emergencial de reconstrução da víscera; além de realização de lavado peritoneal, bem como diálise peritoneal para remoção das toxinas acumuladas em decorrência do uroabdome.

A escolha das suturas de maneira coerente, bem como a realização das mesmas dentro da técnica correta; permitiram a permanência da sonda pelo tempo pretendido, evitando-se a remoção precoce da mesma pelo paciente. Além disso, o conforto gerado ao animal pela redução da distensão abdominal foram fatores determinantes para que o felino não se sentisse incomodado pela sonda.

Devido à iminente possibilidade de ocorrência de novas infecções do trato urinário (ITU), como descrito em um estudo (10), a antibioticoterapia foi empregada durante todo o período no qual o paciente permaneceu sondado, bem como antissepsia da sonda com

Pereira JA, Neves ARP, Oliveira P, Almeida ACO, Soares CS, Figueiredo KBW. Cistostomia com sonda de Foley para desvio temporário do fluxo urinário em felino acometido por ruptura uretral iatrogênica. Vet. e Zootec. 2020.; 2: 001-007. 
clorexidine; cuidados esses que refletiram na não-ocorrência de ITU, mesmo após o procedimento de uretrostomia definitiva.

Em humanos, a técnica de uretrostomia definitiva em porção final de cólon descendente mostra-se bastante eficaz, onde os pacientes permanecem eliminando urina no trato digestório sem ocorrência de complicações. Este mesmo método de uretrostomia foi empregado no paciente em questão, com sucesso, o que implica em estudos futuros do emprego desta técnica, objetivando a utilização com êxito em medicina veterinária para a maior parte dos pacientes.

\section{CONCLUSÃO}

A técnica de cistostomia temporária empregada, associada à terapia de suporte, possibilitou a estabilização do paciente, sendo considerada uma opção efetiva a ser empregada em animais que aguardam a estabilização do quadro clínico antes de procedimentos cirúrgicos definitivos.

\section{REFERÊNCIAS}

1. Corgozinho KB, Souza HJM. Condutas na desobstrução uretral. In: Souza HJM. Coletâneas em medicina e cirurgia felina. Rio de Janeiro: LF Livros de Veterinária; 2003. p.68-88.

2. Reche Junior A, Camozzi RB. Doença do trato urinário inferior dos felinos/cistite intersticial. In: Jericó MM, Andrade Neto JP, Kogika MM. Tratado de medicina interna de cães e gatos. Rio de Janeiro: Rocca; 2015. p.1483-92.

3. Neri AM, Machado LHA, Okamoto PTCG, Filippi MG, Takahira RK, Melchert A, et al. Routine screening examinations in attendance of cats with obstructive lower urinary tract disease. Top Companion Anim Med. 2016;31(4):140-5.

4. Dulaney DR, Hopfensperger M, Malinowsk R, Hauptman J, Kruger JM. Quantification of urine elimination behaviors in cats with a video recording system. J Vet Intern Med. 2017;31(2):486-91.

5. Buffington TCA, Westropp JL, Chew DJ. From FUS to pandora syndrome: where are we, how did we get here, and where to now? J Feline Med Surg. 2014;16(5):385-94.

6. Assis MF, Taffarel MO. Doença do trato urinário inferior dos felinos: abordagem sobre cistite idiopática e urolitíase em gatos. Enciclopedia Biosfera. 2018;15(27):390-404.

7. Corgozinho KB, Souza HJM, Pereira NA, Belchior C, Silva MA, Martins MC, et al. Catheter-induced urethral trauma in cats with urethral obstruction. J Feline Med Surg. 2007;9(6):481-6.

8. Ruda L, Heiene R. Short- and long-term outcome after perineal urethrostomy in 86 cats with feline lower urinary tract disease. J Small Anim Pract. 2012;53(12):693-8.

9. Sævik BK, Trangerud C, Ottesen N, Sørum H, Eggertsdóttir AV. Causes of lower urinary tract disease in Norwegian cats. J Feline Med Surg. 2011;13(6):410-7.

Pereira JA, Neves ARP, Oliveira P, Almeida ACO, Soares CS, Figueiredo KBW. Cistostomia com sonda de Foley para desvio temporário do fluxo urinário em felino acometido por ruptura uretral iatrogênica. Vet. e Zootec. 2020.; 2: 001-007. 
10. Wald HL, Ma A, Bratzler DW, Kramer AM. Indwelling urinary catheter use in the postoperative period: analysis of the national surgical infection prevention project data. Arch Surg. 2008;143(6):551-7.

Recebido em: 20/02/2020

Aceito em: 17/11/2020

Pereira JA, Neves ARP, Oliveira P, Almeida ACO, Soares CS, Figueiredo KBW. Cistostomia com sonda de Foley para desvio temporário do fluxo urinário em felino acometido por ruptura uretral iatrogênica. Vet. e Zootec. 2020.; 2: 001-007. 